\title{
Application of Intelligent Technology Based on Measurement and Control Technology and Instrument
}

\author{
Yu Wang \\ School of Zhejiang University of Technology, Hangzhou 310000, China \\ 468162540@qq.com
}

Keywords: measurement and control technology; instrument; intelligent technology; application

\begin{abstract}
With the rapid development of science and technology, the intelligent technology of measurement and control technology and instrument has made breakthroughs. And the intelligent technology of measurement and control technology and instrument has been widely applied. It has played a positive role in people's production and life. At present, how to effectively play the effectiveness of intelligent technology of measurement and control technology and instrument has become a common research topic of the industry. This paper mainly analyzes the measurement and control technology and the application of the instrument intelligent technology, and then effectively improves the application ability of the measurement and control technology and the intelligent technology of the instrument.
\end{abstract}

\section{Preface}

At present, measurement and control technology has become an important technology in measurement technology in China. Measurement and control technology has been widely applied in industrial production, and remarkable results have been achieved. Fully exerting its application function and liberating the productive forces to a large extent. However, compared with the western developed countries, the Intellectualization Technology of measurement and control technology and instruments still has many deficiencies. Therefore, our country should continue to increase investment in the technology, organize relevant scientific research personnel to carry out research and development, improve their technical functions, in order to promote the sustainable development of China's economy, and provide a good technical basis.

\section{Brief analysis of intelligent technology of measurement and control technology and instrument}

\subsection{Analysis of measurement and control technology}

In the development process of China's industry, the application of measurement and control technology has a positive role in promoting the development of industry and supporting the development of China's industry. Measurement and control technology also plays an important role in improving the market competitiveness of enterprises and improving productivity. At present, China is developing advanced technology industry, and the measurement and control technology has a positive role in promoting the development of high-end technology. However, the measurement and control technology in China is still in the basic stage, and there is still a certain gap compared with the developed countries, and the development is relatively slow. To some extent, the development level of measurement and control technology restricts the development level of China's productive forces, thus restricting the progress of the whole market economy. Based on this, our country should actively draw on the advanced experience of foreign countries and combine the actual development of our country and apply computer technology to the development of measurement and control technology in a timely manner. Thus, the intelligent development of the measurement and control technology is realized. 


\subsection{Intelligent technology analysis of instrument}

In the process of industrial production, advanced instruments symbolize the level of industrial development in the region. Advanced instruments and equipment will accelerate the development of industry. Therefore, some countries attach great importance to the development of instruments. And take it as the strategic goal of giving priority to the development of the country. Therefore, in view of the importance of instrument and instrument, our country should carry out relevant development countermeasures, actively introduce foreign advanced instruments, based on the actual development of current instruments in our country. On the basis of drawing on foreign technology, we should give priority to the development of our own technology, constantly increase investment in science and technology and carry out independent research and development. Constantly enhance the competitiveness of China's market economy. In addition, instruments and meters need to be applied to practical production, so that they can be tested by practice, so as to increase the scientificity of instruments and meters. Constantly improve the intelligent and independent process of instrumentation, so that its better application in concrete production practice.

\section{Intelligent technology and application of measurement and control technology and instrument}

\subsection{Application of intelligent virtualization technology in software development}

In the practical application, intelligent virtual technology realizes human-computer interaction efficiently, and provides a good technical foundation for the development of computer software in the simulation of natural action and sound ${ }^{[1]}$. For example, in terms of grain reserves, efficient control of temperature is needed. In the actual temperature control, if the enterprise adopts the artificial control scheme, it will certainly increase the enterprise running storage cost. If the use of intelligent virtual technology, the development and utilization of storage software, through the interface board, A/D converter, acquisition boards and other use. Then the function of micro computer will be greatly improved. When the storage temperature exceeds the actual control temperature, the system will automatically align to detect, and print the relevant results of the test. At this time, the system will automatically open the ventilator and reduce the internal temperature of the warehouse, so the temperature of the storehouse will be controlled in time.

\subsection{Application in the breeding of silkworm seeds}

In the measurement of the proper temperature and temperature inside the greenhouse, it is necessary to apply the temperature probe sufficiently. The probe is mainly realized by computer. In actual measurement, if the measured humidity of the thermometer does not reach the relevant standards, then the computer will take relevant measures to implement on the basis of a specific $\operatorname{program}^{[2]}$. For example, in the actual temperature measurement, the computer will have related operating procedures according to the change of temperature. When the temperature in the greenhouse is super standard, the computer will automatically open the ventilator and use the ventilator to cool the room. Conversely, when the indoor temperature is relatively low, the computer will automatically start heating equipment, and constantly enhance the indoor temperature.

\subsection{Application in the field of industry and new energy}

In the traditional mode, the measurement work is usually done by contact. The traditional way of measurement will not be disturbed by the external space factors, but it increases the labor intensity to a large extent. In recent years, with the rapid development of science and technology, the non-contact measurement method is gradually replacing the traditional contact measurement method. And a great deal of measuring technology has been developed, especially in material measurement, which has been greatly changed. Material measurement in traditional mode requires the measurement of quartz crystal and mercury vapor. However, at present, infrared measurement can be used to measure it. Infrared measurement can not only improve the accuracy of the measurement results, but also have strong stability. In addition, in the field of new energy, accurate and stable data information can 
improve the production cost of new energy enterprises and enhance reliable data protection.

\subsection{Application of new sensing technology equipment}

With the rapid development of science and technology, the types of measurement and control technology and equipment are relatively various. For example, integrated sensors, digital sensors and so on. The application range of digital sensors is relatively wide. In the actual social production and life, digital sensors are applied to the monitoring system inside the bank, and their performance is also played in different degrees in the environment temperature. The application of integrated sensors is more pressure and visual field measurement. The application of measurement and control technology and instrument in the actual production and life not only promotes the development of technology in the related technical fields, but also plays an active role in promoting the application and development of sensing technology. Therefore, it is of realistic significance to actively develop the utilization value of measurement and control technology.

\subsection{Application in remote measurement and control field}

Remote measurement and control technology has a relatively wide range of applications in the current production and life, and the effective application of remote measurement and control technology has a positive role. The measurement and control technology involves a relatively wide range of content, mainly including wireless communication and control technology, remote measurement and control technology, telephone network measurement and control technology. At present, the application of measurement and control technology is indispensable in oil pipeline dredging and oil transportation. The measurement and control technology plays an important role in the people's production and life. It can not only accurately test the problems existing in the actual operation of water and gas, but also complete the detection work on the related equipment. Therefore, our country should increase the research and development of measurement and control technology, continue to invest funds, strive to create a first-class measurement and control technology, so that it better for our production and life service.

\section{Conclusion}

To sum up, looking at the application and development of various fields of technology in China, the upgrading and upgrading of B will surely promote the improvement of the actual productivity level. The development of intelligent technology of measurement and control technology and instrument is heading for the trend of rapid development. The application of measurement and control technology and intelligent technology in the actual industrial production will certainly promote the improvement of industrial productivity. In addition, in view of the current development of China's industrial field, increasing the research and application of measurement and control technology and intelligent technology has a positive effect on promoting the development of industrialization.

\section{References}

[1] Song Chao. Application Research on Intelligent Technology of measurement and control technology and instrument [J]. Technology innovation and application, 2017, No.19309:292.

[2] Xu Bo. Application of intelligent technology in measurement and control technology and instruments. [J]. era agricultural machinery, 2017, V.44; No.29101:86+88.

[3] Yu Wensong, Qi Zhiwu, Ju Jin Zhu. Application Research on Intelligent Technology of measurement and control technology and instrument [J]. Sichuan cement, 2017, No.24703:150. 